\title{
A difficult art of crystallizing and characterizing elusive polymorphs of meloxicam
}

\author{
J. Śniechowska, P. Paluch, M. K. Dudek \\ Centre of Molecular and Macromolecular Studies, Polish Academy of Sciences, Sienkiewicza 112, 90-363 Lodz, Poland \\ mdudek@cbmm.lodz.pl
}

Meloxicam (MLX), a widely used anti-inflammatory drug, can crystallize as one of four neat polymorphic forms and one hydrated form, as reported in the original MLX patent [1]. However, only its commercially available form I and hydrated form IV have their crystal structure determined and were obtained in their pure forms by other researchers, with forms II, III and V remaining elusive. Recently, having found an admixture of a small amount of form III of MLX in one of the commercially available drug formulations, Freitas et al. attempted to obtain pure form III by repeating crystallization procedures described in the patent, but all trials remained unsuccessful [2].

In this contribution we describe our efforts to obtain and characterize three elusive polymorphs of MLX using Crystal Structure Prediction (CSP) calculations and various crystallization approaches. Each of the three solid forms required a different approach, among which were meticulous and numerous repetitions of examples described in the patent, application of various crystallization additives and gel phase crystallization. Crystal Structure Prediction calculations were exploited in two different ways. First, they were used to indicate the prevalent hydrogen bonding patterns found in energetically favourable crystal structures of MLX in order to rationalize possible crystallization routes. It was found, for example, that form II, identified on the calculated CSP landscape by comparison of the experimental and theoretical powder X-Ray diffractograms, display different hydrogen bonding (HB) pattern than commercially available form I (Figure 1). This led us to perform crystallization experiments using nitrogen-containing heterocycles as additives [3] and gel phase crystallization with a hope to re-direct the crystallization route from form I to the three elusive polymorphs.
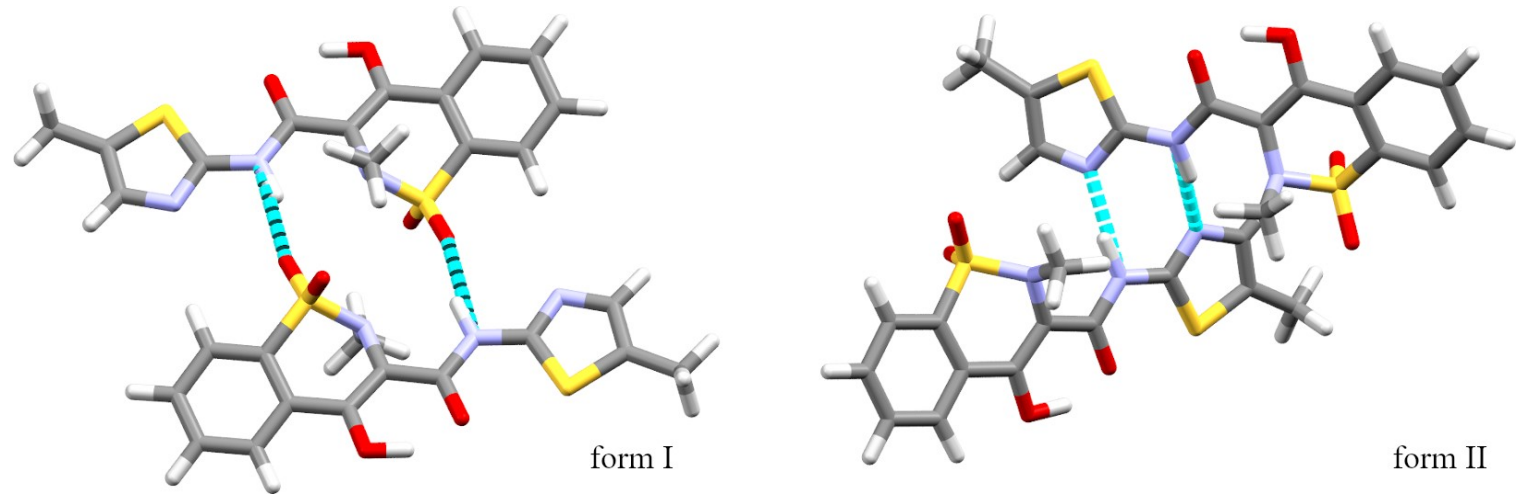

Figure 1. A comparison of HB pattern present in the known form I of MLX and elusive form II found from the CSP calculations.

The second way of exploiting CSP calculations was to characterize the obtained polymorphs of MLX, in combination with NMR crystallography approach. All of the elusive polymorphs of MLX crystallized as microcrystalline powders, which excluded a possibility of characterizing them with single-crystal X-Ray diffraction. Instead, solid-state NMR under very fast magic angle spinning conditions and PXRD diffraction were used to guide the calculations, identify crystal structures of the elusive polymorphs on the CSP landscape, as well as to validate the calculated structures. The presented results are a step towards understanding a fine interplay between the crystal structure and the method of crystallization of elusive polymorphs.

[1] Coppi, L., Sanmarti, M. B. \& Clavo, M. C. (2003). US patent 2003/0109701 A1.

[2] Freitas, J. T. J., Santos Viana, O. M. M., Bonfilio, R. Doriguetto, A. C. \& de Araujo, M. B. (2017). Eur. J. Pharm. Sci. $109,347$.

[3] Thomas, L. H., Wales, C. \& Wilson, C. C. (2016). Chem. Commun. 52, 7372.

Keywords: Crystal Structure Prediction; polymorphism; NMR crystallography; methods of crystallization; meloxicam

This work was financially supported by Polish National Science Center (UMO-2018/31/D/ST4/01995). PL-GRID is is gratefully acknowledged for providing computational resources. 